\title{
A PROJECT FOR INVESTIGATION OF THE STELLAR POPULATIONS OF THE GALACTIC DISK
}

\author{
V. STRAIŽYS \\ Institute of Theoretical Physics and Astronomy \\ Goštauto 12, Vilnius 2600, Lithuania
}

AND

A. G. D. PHILIP

Institute for Space Observations

1125 Oxford Pl., Schenectady, NY 12308, U.S.A.

All models of the visual Galaxy are verified only in fields with galactic latitude $|\mathrm{b}|>20^{\circ}$. At lower galactic latitudes, all the broad-band photometric systems and the majority of medium-band photometric systems fail to classify stars due to interstellar reddening. For the Vilnius photometric system the interstellar reddening is not an obstacle.

The Vilnius system contains seven passbands at 345, 374, 405, 466, 516, 544 and $656 \mathrm{~nm}$ with half-widths of the order of $20 \mathrm{~nm}$. It allows one to obtain spectral types, luminosities, metallicities and the interstellar reddening of stars completely photometrically. The system is especially effective when used with CCD detectors which combine a wide field, high sensitivity and high photometric accuracy. This makes it possible to classify very faint and distant stars in those Milky Way areas affected by variable amounts of interstellar reddening. No other photometric system currently in use, can do this type of work.

A program for investigation of the stellar population in the galactic disk is proposed. It is based on CCD seven-color photometry of stars down to 18th mag in 25 fields of $20^{\prime} \times 20^{\prime}$ size near the galactic plane in directions where interstellar extinction is low. It is estimated that each area will contain about $2000-5000$ stars.

Spectral types, luminosity classes, absolute magnitudes, metallicities, interstellar reddenings and distances for all the stars in the areas will be determined. These data can be used to obtain space densities of different types of stars, luminosity functions, vertical scale height, radial scale length and thick disk parameters in different directions in the galactic plane, including spiral arms, interarm regions and the central bulge. This information can be used to verify the existing models of the Galaxy (or to propose new ones) near its plane.

The full text of the paper will be published in Baltic Astronomy, vol.3, No. 1/2, p. 146-157, 1994. 